DOI: $10.1007 /$ s10357-012-2350-9

\section{Handbuch des Technikrechts}

Martin Schulte/Rainer Schröder (Hrsg.), Handbuch des Technikrechts. Springer-Verlag. 2. Auflage 2011, XXXVI, 940 Seiten, Berlin/Heidelberg.

Das Rechtsgebiet „Technikrecht“ ist ein in der Fachliteratur (zu Unrecht) noch selten zu findender Begriff, lassen sich unter dieser Sammelbezeichnung doch nur zu gut zahlreiche technikbezogene Rechtsgebiete zusammenfassen, deren Verwandtschaft der Materie und Rechtssystematik nach doch zumeist klar zu erkennen ist (Man denke bei der thematischen Schwerpunktsetzung auch nur an das seit nunmehr fast zweieinhalb Jahrzehnten existierende renommierte Institut für Umwelt- und Technikrecht an der Universität Trier.). Weitergehend als das bisweilen zu findende Umwelt- und Technikrecht, das seinen Fokus auf das umweltbezogene Technikrecht unter Hinzunahme des Umweltplanungsrechts bezieht, verstehen die Herausgeber des vorliegenden Werks, dessen Erscheinen nach der Erstauflage im Jahre 2003 nunmehr bereits in zweiter Auflage anzuzeigen ist, unter dem Begriff „Technikrecht“ eine Querschnittsmaterie bestehend u.a. aus Umwelt-, Energie-, Telekommunikations-, Produktsicherheits-, Patent- und Computerrecht sowie weiteren Gebieten mit Technikbezug.

Das Handbuch ist inhaltlich unterteilt in einen Allgemeinen und einen Besonderen Teil. Während sich der Allgemeine Teil sich mit übergreifenden Themen im Kontext des Technikrechts beschäftigt, umfasst der Besondere Teil eine Auswahl spezialgesetzlich geregelter Bereiche mit besonderem Technikbezug. Der Autorenkreis setzt sich wie in der ersten Auflage aus einem Kreis renommierter Wissenschaftlerinnen und Wissenschaftler aus dem Hochschulsektor zusammen.

Der Allgemeine Teil beginnt zunächst mit einer sehr lesenswerten „Kurzen Geschichte des Technikrechts“ von Miloś Vec vom Frankfurter Max-Planck-Institut für europäische Rechtsgeschichte, die dem Leser historisch in die Materie einleitend die Entstehung und der daran anknüpfenden Verrechtlichung des Technikrechts vor Augen führt. Es schließt sich ein kurzes von Jost Halfmann, Inhaber einer Professur für Techniksoziologie an der TU Dresden, verfasste Kapitel zu „Technikrecht aus Sicht der Soziologie“ an, gefolgt von einem Kapitel „Technikrecht und Ökonomische Analyse“ von Peter Salje, in dem es um die ökonomischen Implikationen technikbezogener Rechtsnormen geht. Das folgende von Michael Kloepfer verfasste Kapitel zu den „Instrumenten des Technikrechts“ stellt dem Leser sodann die nach Rechtscharakter und Typologie zu unterscheidenden Normen (u.a. auch die in praxi äußerst bedeutsamen Techniknormen) und Steuerungsmechanismen dar. Die folgenden beiden Kapitel von Anne Röthel von der Hamburger Bucerius Law School und Herausgeber Rainer Schröder befassen sich mit den Europarechtlichen Vorgaben (Röthel) bzw. den Verfassungsrechtlichen Rahmenbedingungen (Schröder) des Technikrechts, wobei insbesondere erstere wegen zahlreicher direkt geltender Normen für den Anwender auch von unmittelbarer Bedeutung ist. Es schließen sich ein weitere von Peter Salje verfasstes Kapitel zum „Anlagenhaftungsrecht“, das den Fokus auf die Haftung von Anlagenbetreibern für ihre Anlagen legt, sowie ein Kapitel zu dem davon zu unterscheidenden „Produkthaftungsrecht" von Klaus Vieweg an, das die zivilrechtliche Haftung für Produkte nach Deliktsrecht und Produkthaftungsgesetz darstellt. Der Allgemeine Teil wird abgeschlossen durch einen Beitrag zum „Technikstrafrecht“ von Irini E. Vassilaki.

Der Besondere Teil beginnt mit einem Beitrag zum ,Gerätesicherheitsrecht" von Franz-Josef Peine, der als Grundlage das Geräte- und Produktsicherheitsgesetz vom 6.1.2004 hat, das mittlerweile von

Rechtsanwalt Dr. Timo Hohmuth,

Grenius Rechtsanwälte, Hamburg, Deutschland dem am 1.12.2011 in Kraft getretenen Produktsicherheitsgesetz abgelöst worden ist. Diese europarechtlich bedingte Novelle hat Veränderungen gebracht, die leider nicht mehr Eingang finden konnten. Es folgt ein Kapitel zu dem Themenkreis „Umwelt und Technik“ von Hellmuth Schulze-Fielitz, das dem Leser einen guten Überblick gewährt, aber angesichts der Fülle von technikbezogenen Normen aus dem Umweltrecht und der zentralen Stellung des technikbezogenen Umweltrechts vielleicht eine gegenüber anderen Teilkapiteln umfassendere Darstellung mit Blick auf einzelnen Teilrechtsgebiete gerechtfertigt hätte. Im darauffolgenden Kapitel zum „Recht der Umwelt- und Humangenetik" geben der Mitherausgeber Martin Schulte und Co-Autor David Apel einen Überblick über das Gentechnikrecht nach dem Gentechnikgesetz, aber auch rechtliche Aspekte des Themenkreises wie z.B. Embryonenschutz oder Stammzellenforschung. Daran schließt sich ein in die Zweitauflage neu aufgenommenes Kapitel zum „Energierecht“ von Ulrich Büdenbender an. Auch dieses Kapitel gibt gemessen an den Dimensionen des Energierechts lediglich einen ersten Überblick über das Rechtsgebiet, das infolge des Atomausstiegs und der klimapolitischen Energiewende neuerlich stark in den Fokus der öffentlichen Beachtung getreten ist. Das im darauffolgenden Beitrag abgehandelte ,Telekommunikations- und Medienrecht als Technikrecht" von Martin Eifert und Wolfgang Hoffmann-Riem trägt seine thematische Begrenzung bereits im Titel und widmet sich v.a. den technikbezogenen Vorgaben des Telekommunikationsrechts sowie dem technischen Medienrecht. Einen äußerst gelungenen Überblick über die „Grundlagen des Patentrechts“ liefern die Autoren Horst-Peter Götting und Heike Röder-Hitschke in dem darauffolgenden Kapitel, bei dem neben dem umfassend umrissenen Patentrecht auch die Grundzüge des Gebrauchsmusterrechts Berücksichtigung finden. Das folgende Kapitel zum „Computerrecht“ von Thomas Dreier vom Zentrum für angewandte Rechtswissenschaften vom Karslruher Institut für Technologie und Oliver Meyer-van Raay fasst wiederum ein nicht zentral normiertes Rechtsgebiet bestehend aus zahlreichen Quellen zusammen, die sich mit der Materie Software, Datenbanken, Computervertragsrecht etc. befassen. Das folgende Kapitel von Hannes Federrath, Lehrstuhlinhaber für Wirtschaftsinformatik an der Universität Regensburg, und seines 2010 verstorbenen Lehrers Andreas Pfitzmann knüpft thematisch an das vorangehende an und beleuchtet das Themenfeld „Datensicherheit“, das auf den systembezogenen Schutz von Daten bezogen abzugrenzen ist vom Datenschutz, der sich auf das staatlich gewährte Recht von Personen auf Schutz ihrer Daten bezieht. Das an dieser Stelle zu erwartende Kapitel zum „,Datenschutz“ fehlt im vorliegenden Werk gegenüber der Vorauflage bedauerlicherweise, wobei es nur aufgrund eines nicht gelieferten Manuskripts entfallen ist, wie man dem Vorwort entnehmen kann. Den Abschluss des Besonderen Teils bildet sodann ein Beitrag von Alexander Roßnagel zur „Rechtsverbindlichen Telekooperation“, in dem übergreifend die Möglichkeiten und Formen rechtsverbindlicher elektronischer Kommunikation (im Signaturgesetz oder spezialgesetzlich) dargestellt werden.

Zusammenfassend lässt sich sagen, dass die Herausgeber bereits in zweiter Auflage ein überaus bemerkenswertes Werk vorlegen, das unter dem Begriff „Technikrecht“ eine Materie verschiedener technikbezogener Rechtsgebiete treffend zu einer Querschnittsthematik zusammenfasst, die die Auseinandersetzung des Normadressaten mit Technik in verschiedenen Lebens- und Rechtsbereichen beschreiben. Das Themenfeld ließe sich sicher noch erweitern (z.B. um Lebensmittel-, Umwelt-, Pharma- und Medizintechnik) und bestimmte bereits aufgegriffene Themenkomplexe noch in größerer Tiefe und Breite darstellen, aber die Konzeption und Themenauswahl überzeugt schon jetzt. Zudem haben sich jeweils führende Wissenschaftler gefunden, die eine gelungene und fachgerechte Darstellung gewährleisten. Der „Handbuch-Charakter“ des Werks wird zudem der möglicherweise zuweilen noch ungewohnt empfundenen Begrifflichkeit des Technikrechts gerecht und sollte gleichsam als ausreichender Auftakt dafür dienen, dieser Zusammenfassung von technikbezogenen Themenkreisen als „Technikrecht“ eine weitergehende Begriffsprägung zu geben. 